\title{
UNIFICATION OF COMMUTATIVE RELATIVITY AND NONCOMMUTATIVE QUANTUM DYNAMICS VIA CYCLIC GROUPOIDS AND SPACETIME FUSION CATEGORIES
}

\author{
Arturo Tozzi (Corresponding Author) \\ Center for Nonlinear Science, University of North Texas \\ 1155 Union Circle, \#311427Denton, TX 76203-5017 USA \\ Computational Intelligence Laboratory, University of Manitoba, Winnipeg, Canada \\ Winnipeg R3T 5V6 Manitoba \\ tozziarturo@libero.it
}

James F. Peters

Department of Electrical and Computer Engineering, University of Manitoba

75A Chancellor's Circle Winnipeg, MB R3T 5V6 CANADA and

Department of Mathematics, Adıyaman University, 02040 Adiyaman, Turkey

James.Peters3@umanitoba.ca

The unexploited unification of general relativity and quantum physics is a painstaking issue that prevents physicists to properly understanding the whole of Nature. Here we propose a pure mathematical approach that introduces the problem in terms of group theory. Indeed, we build a cyclic groupoid (a nonempty set with a binary operation defined on it) that encompasses both the theories as subsets, making it possible to join together two of their most dissimilar experimental results, i.e., the commutativity detectable in our macroscopic relativistic world and the noncommutativity detectable in the quantum, microscopic world. This approach, combined with the Connes fusion operator, leads to a mathematical framework useful in the investigation of relativity/quantum mechanics relationships.

\author{
KEYWORDS: \\ Einstein; manifold; Hilbert space; Abelian; dagger category
}

Both general relativity (GR) and quantum physics (QF) describe Nature using mathematical structures such as tensors and probability theory (Yilmaz, 1982; Comte, 1996; Fre, 2013). The unification of GR and QF stands for the Holy Grail in physics: indeed, the two issues, despite the fact that both work very well in the description of different features of Nature, seem to be incomparable and not reducible to a unified, self-consistent framework. Several recent efforts have been provided, in order to correlate them (e.g., Ghose, 1997; Skalsky, 2010; Fre, 2013; Elitzur, Dolev, Kolenda, 2017; Aerts, 2017), but they tend to paint a somewhat incomplete picture of the milieu described by GR and QF. Indeed, quantum dynamics and special relativity display very different behaviours and features, starting from the very subtending mathematical structures. General relativity is described on a 3+1-dimensional pseudo-Riemannian manifold with tensor fields obeying certain partial differential equations, while quantum field theory is described on an $\mathrm{R}^{4}$ projective Hilbert space, with operator-valued fields obeying certain Lorentz-invariant partial differential equations and commutation relationships (Tegmark 2008). Therefore, in the Euclidean framework, describable by three spatial dimensions plus time, we achieve vectors equipped with just one basis, while in the projective Hilbert space of quantum mechanics, equipped with infinite dimensions, we achieve vectors with different bases which are sets of equivalence classes. In other words, the macroscopic world of relativity has an orthonormal basis common to all the observables, i.e., a set of vectors $B$ in Euclidean or Hilbert space such that every vector can be written as a linear combination of vectors from B, while all vectors from B have length 1 and any two of them are orthogonal (Tumulka, 2009). On the other side, the microscopic world of quantum dynamics displays different bases that are the source of the rather counterintuitive physical effects experienced during experimental observations.

Efforts have been provided in order to correlate the two different manifolds. For example, techniques of deformation quantization, such as the Wigner-Weyl transform, have provided rules in order to strain the "classical" commutative algebra of observables into a quantum noncommutative one (Curtright et al., 2014). In this paper we will focus, rather than on manifolds, on another difference between the two physical frameworks of GR and QF: the commutability (Goodman, 2003). In the macroscopic world dictated by GR, the binary operation of multiplication is commutative, i.e., changing the order of the operands does not change the result. This means that: $a b=b a$. On the other side, in QF, the linear operators representing a pair of physical variables do not commute, rather are mutually complementary. This means that $c d \neq d c$. Examples of complementary quantum properties which cannot all be observed or measured simultaneously are position and momentum, energy and duration, spin on different axes, wave and particle, entanglement and coherence, and so on (Kalckar et al., 1996). Here we raise the question whether it is feasible to correlate the two 
mathematical operations of commutativity and noncommutativity in a unified scheme. Such issue (a set that encompasses both the commutativity and noncommutativity subsets) cannot be tackled through the otherwise successful FraenkelZermelo set, because some of its axioms cannot work properly in this peculiar context. Therefore, we ask here: is there a loop between commutativity and noncommutativity, that gives rise to a quantifiable graph?

\section{A CYCLIC GROUPOID ENCOMPASSES LOOPS BETWEEN COMMUTATIVE AND NONCOMMUTATIVE OPERATIONS}

We start from a groupoid A, equipped with a commutative binary operation, and a groupoid B, equipped with a noncommutative binary one. We require that a single observable must be either macroscopic (commutative, subjected to relativity) or microscopic (noncommutative, subjected to quantum dynamics). Here we show that a set $\mathrm{C}$ does exist, able to encompass both A and B. The procedure is illustrated in Figure 1.

The set $\mathrm{A}=\{\mathrm{a}, \mathrm{b}, \mathrm{c}\}$, while the operation $\langle 0\rangle: \mathrm{AxA} \rightarrow \mathrm{A}$.

$\mathrm{A}<\mathrm{O}>\mathrm{b}=\mathrm{b}<\mathrm{O}>\mathrm{a}=\mathrm{b}$ reads: "( $\mathrm{a}, \mathrm{b})$ maps to $\mathrm{b}$ ", and "( $\mathrm{b}, \mathrm{a})$ maps to $\mathrm{b}$ ".

In general, $\mathrm{x}<0>\mathrm{y}=\mathrm{y}<\mathrm{0}>\mathrm{X}$ for all elements $\mathrm{x}, \mathrm{y}$ in $\mathrm{A}$. This means that $(\mathrm{A},<\mathrm{O}>)$ is an Abelian groupoid, and the operation o is commutative.

The set $\mathrm{B}=\{\mathrm{d}, \mathrm{e}, \mathrm{f}, \mathrm{g}\}$, while the operation $\langle\mathrm{o}\rangle: \mathrm{BxB} \rightarrow \mathrm{B}$.

$\mathrm{d}<\mathrm{o}>\mathrm{e}=\mathrm{e}$ reads: "(d,e) maps to e", and "(e,d) maps to e". Further, e <o $>\mathrm{f}=\mathrm{f}$ reads: "(e,f) maps to f", but $\mathrm{f}<\mathrm{o}>\mathrm{e}=\mathrm{f}$ does not map to $\mathrm{f}$ ".

This means that $(\mathrm{B},<\mathrm{O}>)$ is a non-Abelian groupoid, and the operation $<0>$ is not commutative.

Sets $\mathrm{A}$ and $\mathrm{B}$ are contained in $\mathrm{C}$. The set $\mathrm{C}=\{\mathrm{a}, \mathrm{b}, \mathrm{c}, \mathrm{d}, \mathrm{e}, \mathrm{f}, \mathrm{g}\}$, while the operation $[\langle\mathrm{o}\rangle]$ : $\mathrm{CxC} \rightarrow \mathrm{C}$.

$\mathrm{a}[<\mathrm{O}\rangle] \mathrm{b}=\mathrm{b}[<\mathrm{O}\rangle] \mathrm{a}=\mathrm{b}$ commutes.

$\mathrm{a}[\langle\mathrm{O}\rangle] \mathrm{d}=\mathrm{d}[\langle\mathrm{O}\rangle] \mathrm{a}=\mathrm{d}$ commutes.

$\mathrm{b}[\langle\mathrm{O}\rangle] \mathrm{e}=\mathrm{e}[\langle\mathrm{O}\rangle] \mathrm{b}=\mathrm{e}$ commutes.

$\mathrm{c}[\langle\mathrm{O}\rangle] \mathrm{f}=\mathrm{f}[\langle\mathrm{O}\rangle] \mathrm{c}=\mathrm{f}$ commutes.

e $[\langle\mathrm{O}\rangle] \mathrm{f}=\mathrm{f}, \mathrm{f}[\langle\mathrm{o}\rangle] \mathrm{e}=\mathrm{e}$ does not commute, and so on.

Therefore, $(\mathrm{C},[<\mathrm{O}>])$ is a non-Abelian groupoid, and the operation $[<\mathrm{O}>]$ is not commutative. 


\section{Set A}

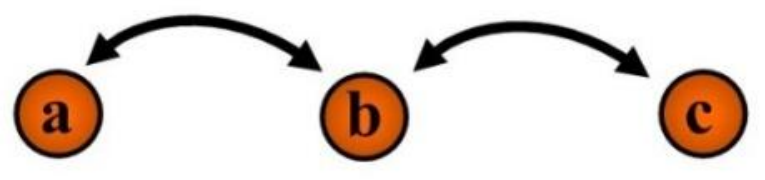

\section{Set B}

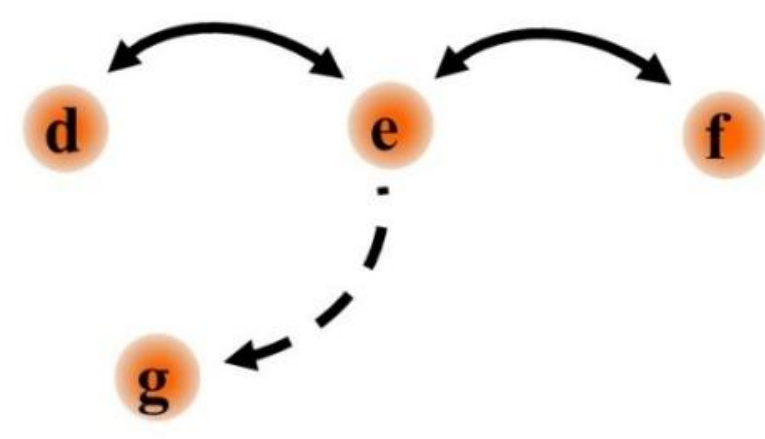

\section{Set C}

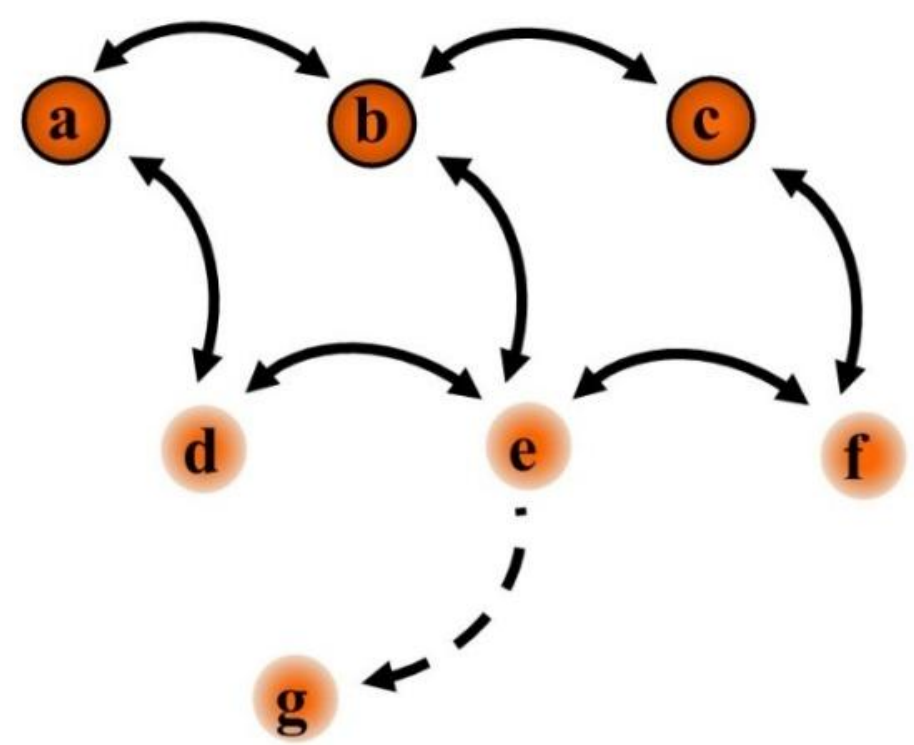

Figure 1. Three sets in terms of cyclic groupoids. The solid, double-arrowed lines stand for commutative relationships, while the dotted arrows for noncommutative ones. The circles $a, b$ and c (encompassed in the set A) stand for physical observables experimentally detectable in our macroscopic relativistic world, equipped with commutative binary operation properties. The circles d, e, f and $g$ (encompassed in the set B) stand for physical observables experimentally detectable in our microscopic quantum world, equipped with noncommutative binary operation properties. The set $\mathrm{C}$ embeds both the sets $\mathrm{A}$ and $\mathrm{B}$. 


\section{A MATHEMATICAL UNIFICATION VIA CYCLIC GROUPOIDS AND SPACETIME FUSION CATEGORIES.}

This section briefly introduces the unification of Abelian and non-Abelian cyclic groupoids in a fusion category. Fusion categories are introduced in Henriques and Penneys (2016) using the Connes fusion operator $\bigotimes$ (Connes, 1994; Bartels, A., Douglas, C.L., Henriques, A. 2014), elaborated in Wassermann (1998).

Let $A_{\circ} \equiv(A, \circ), B \circ \equiv(B, \circ)$ denote a pair of cyclic groupoids, each with a binary operation o.

Let $[x]$ denote a Connes fusion operator, which is an associative product on bimodules (in our case, a bimodule is the Cartesian product of a pair of cyclic groups) and let $g: A_{\circ} \times B_{\circ} \rightarrow A_{\circ}[\times] B_{\circ}$ be an isomorphishm on a pair of cyclic groupoids onto the fusion of the groupoids $A_{\circ}, B_{\circ}$. The fusion of these groupoids is represented in Figure 2. Putting this together, we obtain

$$
\left(A_{\circ}, B_{\circ}\right) \stackrel{g}{\mapsto} A_{\circ}[\times] B_{\circ} \mapsto A_{\circ} \cup B_{\circ}=(A \cup B, \circ) .
$$

Thanks to the Connes fusion operator, we obtain a mathematical framework in the form of a fusion category, which is useful in reasoning about the unification of quantum theory and relativity. A fusion category consists of a collection of objects and a collection of morphisms (maps between objects in a fusion category). The focus here is on fusion categories in which the objects are cyclic groupoids and associative products (fused) bimodules, while the morphisms are mappings on pairs of cyclic groupoids or fused cyclic groupoids.
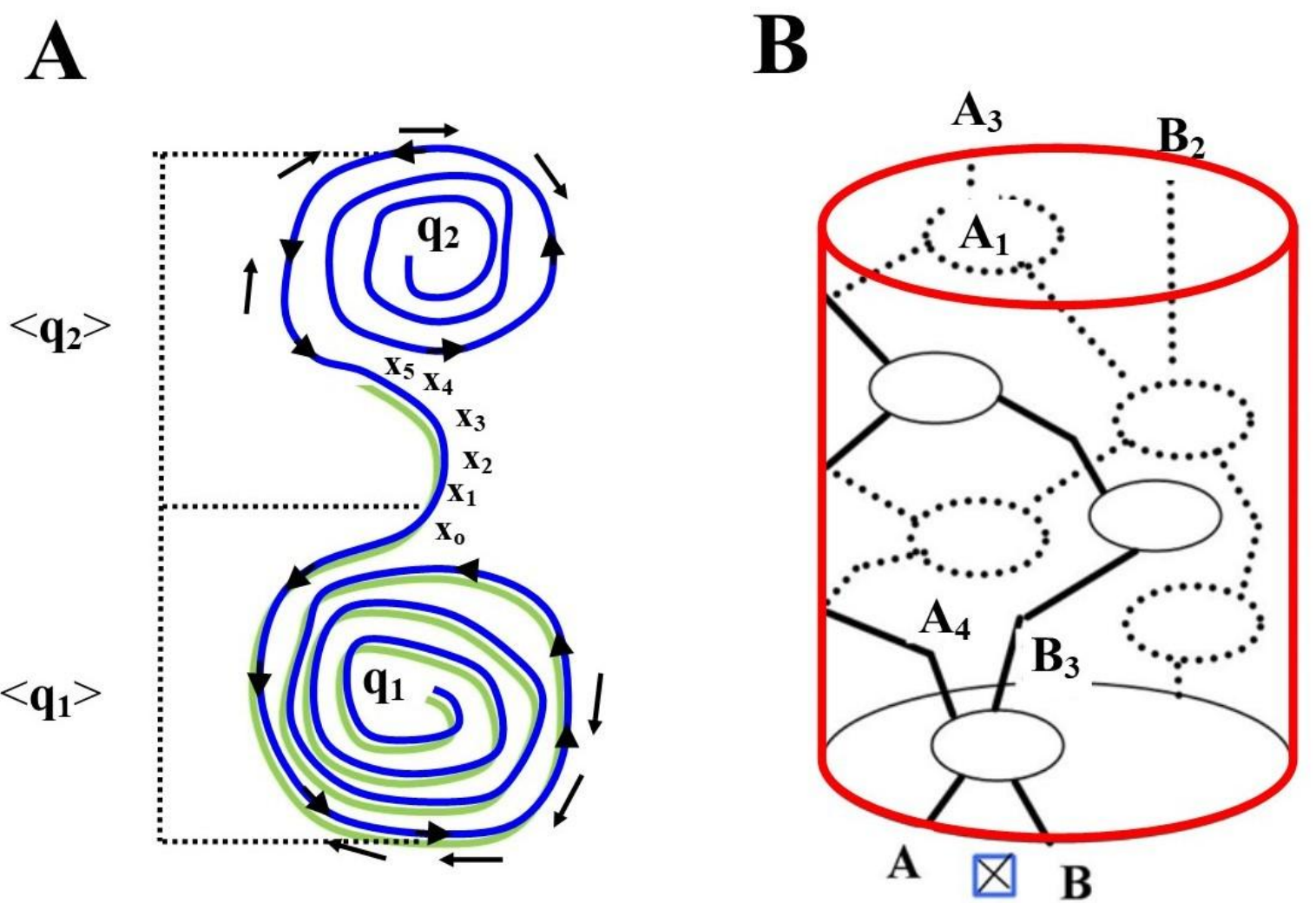

Figure 2A: Generators for a Cyclic Groupoid. The cyclic groupoid $A_{\circ}$ has a generator $\left\langle q_{1}\right\rangle$. Similarly, the cyclic groupoid $B_{\circ}$ has a generator $\left\langle q_{2}\right\rangle$. This means, for example, each element $A_{i}=q_{1} \circ 2 q_{1} \cdots \circ k q_{1}$ in $A_{\circ}$ is a linear combination of the generator $q_{1}$. The fusion of the two groupoids results in a new groupoid, namely, $A_{\circ} \cup B_{\circ}=(A \cup B, \circ)$.

Figure 2B: Spacetime View of a Fusion Category. A spacetime view of a fusion category is shown in Fig. X(b). Over time, 


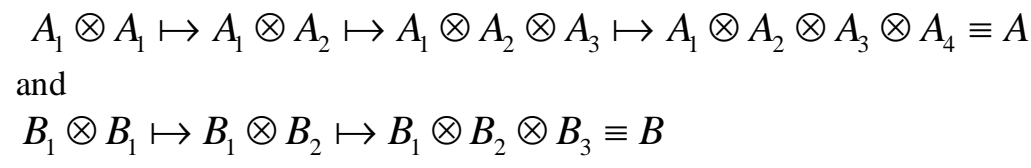

which are Connes fused, resulting in $A[\times] B$, that is an object in the spacetime fusion category.

\section{CONCLUSIONS}

Several efforts have been made in order to unify GR and QT, but, until now, the results are missing. Many investigations aimed to provide transformation rules between the two manifolds of GR and QT. To make an example, Feffman et al. (2015) assessed the geometric Whitney problem on how a Riemannian manifold can be constructed to approximate a metric space. Deformation quantization (such as the above-mentioned Wigner-Weyl transform) is able to achieve a complete phase space formulation of quantum mechanics, equivalent to the Hilbert-space operator representation, with star-multiplications that are isomorphically parallel operator multiplications (Curtright et al., 2014). By expressing quantum mechanics in the same phase space as that of classical mechanics, the map achieved through the Wigner-Weyl transform facilitates recognition of quantum mechanics as a deformation of the classical one. However, such techniques are limited to the description of just a mere representation change from Hilbert space to phase space, because they are not able to build a successful quantization scheme, namely a method to produce a quantum theory out of a classical one. Furthermore, very last experimental efforts point towards the possibility of simultaneous observation of quantization and interference patterns (Piazza et al., 2015). However, the latter experiment stands for a technical device, rather than a proper conceptual framework able to unify GR and QT.

Special relativity has been tackled also by the noncommutative standpoint (Besnard and Bizi, 2017). Girelli and Livine (2004) argued that Deformed Special Relativity is obtained by imposing a maximal energy to Special Relativity and by deforming the Poincare symmetry in order to accommodate this requirement. Variations of the same procedure lead to a noncommutative space structure that preserves conservation laws. In turn, the Very Special Relativity's (VSR) approach demonstrated that that the subgroup of the Poincare group is sufficient to describe the spacetime symmetries of observed physical phenomena. Indeed, Das et al. (2011) introduced a novel noncommutative spacetime structure that enjoys the symmetries of deformed VSR: this trick allowed them to build a point particle Lagrangian that lives in a noncommutative phase space. Some Authors view QT as a deformation of the combinatorial or Hamiltonian quantization of threedimensional gravity in the Chern-Simons formulation. In this approach, quantum groups replace the local isometry groups, and noncommutative spacetimes replace the classical model spacetimes (Schroers, 2011). Furthermore, Kalau and Malze (1995) derived an action for gravity in the framework of noncommutative geometry by using the Wodzicki residue. Furthermore, they achieved a gravity action for commutative geometry which is the usual Einstein-Hilbert action. They also provided a noncommutative extension given by the tensor product of the algebra of smooth functions on a manifold and a finite dimensional matrix algebra.

In contrast with the above-mentioned approaches, here we provide a purely mathematical framework, where the unification of the symplectic phase space of the macroscopic relativistic world and the noncommutative phase space of the microscopic quantum world it is not strictly required. Indeed, our mathematical approach allows to join, in a single group theoretic framework, both the commutative and noncommutative operations that stand for, respectively, the macroscopic relativistic world and the microscopic quantum realm.

Our framework paves the way to fully mathematical approaches which unify quantum dynamics and general relativity. Another possibility, not explored here, is to use of an involutive functor between the opposite categories of commutativity and noncommutativity, in order to attain a dagger structure with the adjoint of a morphism being the inverse. This treatment would make it possible to achieve morphisms that are unitary in finite dimensional Hilbert spaces, in order to build a dagger symmetric monoidal category (Blute and Panangaden, 2011) which is also compact closed, resulting in completeness results that extend the use of Hilbert spaces to relativity issues. To accomplish a dagger category, a fusion category is equipped with a dagger $\dagger$ so that morphisms $f: A \rightarrow B$ have a two-side inverse (Huenen and Karvoven, 2016).

\section{REFERENCES}

1) Aerts, D.; Aerts, S. 2017. Towards a general operational and realistic framework for Quantum mechanics and Relativity Theory, in A. Elitzur, S. Dolev, N. Kolenda, Eds., Quo Vadis Quantum Mechanics, Springer, 2017. 
2) Bartels, A., Douglas, C.L., Henriques, A. 2014. Dualizability and index of subfactors. arXiv 1110.5671v2,136.

3) Besnard F, Bizi N. 2017. On the definition of spacetimes in Noncommutative Geometry: Part I. arXiv:1611.07830v3 [math.OA] 13 Mar 2017

4) Blute, R., Panangaden, P. 2011. Dagger categories and formal distributions. Lecture Notes in Physics 813, Springer, Heidelberg, 421-436, DOI 10.1007/978-3-642-12821-9_6, MR2767050.

5) Connes, A. 1994. Noncommutative Geometry. Academic Press Inc., San Diego, CA.

6) Curtright, T. L.; Fairlie, D. B.; Zachos, C. K. 2014. A Concise Treatise on Quantum Mechanics in Phase Space. World Scientific. ISBN 9789814520430.

7) Comte C. 1996. Symmetry, relativity and quantum mechanics, Il Nuovo Cimento, vol. 111 B, no. 8, 937-956.

8) Das S, Ghosh S, Mignemi S. 2011. Non-commutative spacetime in very special relativity. Physics Letters A. Volume 375, Issue 37, 29 August 2011, Pages 3237-3242. https://doi.org/10.1016/j.physleta.2011.07.024.

9) Elitzur, A.; Dolev, S.; N. Kolenda, Eds. 2017. Quo Vadis Quantum Mechanics, Springer.

10) Fefferman C, Ivanov S, Kurylev Y, Lassas M, Narayanan H. 2015. Reconstruction and interpolation of manifolds I: The geometric Whitney problem. arXiv:1508.00674

11) Fré, P.G. 2013. Gravity, a Geometrical Course. Vol. 1: Development of the Theory and Basic Physical Applications, Springer, DOI 10.1007/978-94-007-5361-7

12) Ghose, P. 1997. Violatioj of signal locality and unitarity in a merger of quantum mechanics and general relativity, Pramana 49, no. 1, 65-69.

13) Girelli F, Livine ER. 2004. Special relativity as a non commutative geometry: Lessons for deformed special relativity. Phys.Rev. D81 (2010) 085041. DOI: 10.1103/PhysRevD.81.085041

14) Goodman F. 2003. Algebra: Abstract and Concrete, Stressing Symmetry, 2e. Prentice Hall. ISBN 0-13067342-0.

15) Henriques, A. and Penneys, D. 2016. Bicommutant categories from fusion categories. arXiv 1511.05226v2,1-35.

16) Heuen, C., Karvoven, M. 2016. Monads on dagger categories. Theory and Applications of Categories, 31 , no. 35, 1016-1043, MR3584697.

17) Kalau W, Malze M. 1995. Gravity, non-commutative geometry and the Wodzicki residue. Journal of Geometry and Physics, Volume 16, Issue 4, July 1995, Pages 327-344. https://doi.org/10.1016/03930440(94)00032

18) Kalckar J, Bohr N, Rosenfeld L, Rüdinger E, Aaserud F. 1996. Foundations of Quantum Physics II (19331958). Elsevier. p. 210. ISBN 978-0-444-89892-0. Retrieved 2011-10-24.

19) Piazza L, Lummen TT, Quiñonez E, Murooka Y, Reed BW, Barwick B, Carbone F. 2015. Simultaneous observation of the quantization and the interference pattern of a plasmonic near-field. Nat Commun. $2015 \mathrm{Mar}$ 2;6:6407. doi: 10.1038/ncomms7407.

20) Schroers BJ. 2011. Quantum gravity and non-commutative space-times in three dimensions: a unified approach. Acta Physica Polonica B Proceedings. Vol. 4 (2011) Supplement No 3

21) Skalsky, V. 2010. The model of a flat (Euclidean) expansive homogeneous and isotropic relativistic universe in the light of the general relativity, quantum mechanics, and observations. Astrophys. Space Sci. 330, 373398, DOI 10.1007/s10509-010-0409-8

22) Tegmark M. 2008. The Mathematical Universe. Foundations of Physics. February 2008, Volume 38, Issue 2, pp 101-150.

23) Tumulka, R. 2009. Orthonormal basis. In: D. Greenberger, K. Hentschel, F. Weinert, eds., Compendium of Quantum Physics, Springer, Berlin, https://doi-org.uml.idm.oclc.org/10.1007/978-3-540-70626-7_135.

24) Yilmaz, H. 1981. Relativity and quantum mechanics, Int. J. of Theoretical Physics 21, no. 10/11, 871-902. 
Hogan C, Kwon O. 2017. Models of Exotic Interferometer Cross-Correlations in Emergent Space-Time. arXiv: $1711.05514 v 4$ [gr-qc] 17 Dec 2017

A Lorentz invariant framework is developed to interpret an exotic cross spectrum in the signals of two separate interferometers, associated with the emergence of space-time and inertial frames from a Planck scale quantum system. Space-time relationships between world lines are modeled as antisymmetric cross-correlations on past and future light cones between sequences in proper time with Planck bandwidth, arising from nonlocal entanglement between geometrical states. Causal diamonds in a at space-time are normalized to have the same holographic information content as black hole event horizons. Models of interferometer response are shown to produce a unique signature: a mostly imaginary broad band cross spectrum, with a frequency structure determined by the layout of the apparatus. The framework will be used for interpreting data from the reconfigured Fermilab Holometer, and for conceptual design of future experiments.

Calmet X, Fritz C. 2015. Inflation on a Non-Commutative Space-Time. arXiv:1506.04049v1 [hep-th] 12 Jun 2015 We study inflation on a non-commutative space-time within the framework of enveloping algebra approach which allows for a consistent formulation of general relatività and of the standard model of particle physics. We show that within this framework, the effects of the non-commutativity of spacetime are very subtle. The dominant effect comes from contributions to the process of structure formation. We describe the bound relevant to this class of non-commutative theories and derive the tightest bound to date of the value of the non-commutative scale within this framework. Assuming that inflation took place, we get a model independent.

Besnard F, Bizi N. 2017. On the definition of spacetimes in Noncommutative Geometry: Part I. arXiv:1611.07830v3 [math.OA] 13 Mar 2017

In this two-part paper we propose an extension of Connes' notion of even spectral triple to the Lorentzian setting. This extension, which we call a spectral spacetime, is discussed in part II where several natural examples are given which are not covered by the previous approaches to the problem. Part I only deals with the commutative and continuous case of a manifold. It contains all the necessary material for the generalization to come in part II, namely the characterization of the signature of the metric in terms of a time-orientation 1-form and a natural Krein product on spinor fields. It turns out that all the data available in Noncommutative Geometry (the algebra of functions, the Krein space of spinor fields, the representation of the algebra on it, the Dirac operator, charge conjugation and chirality), but nothing more, play a role in this characterization. Thus, only space and time oriented spin manifolds of even dimension are considered for a noncommutative generalization in this approach. We observe that these are precisely the kind of manifolds on which the modern theories of spacetime and matter are defined. 